HNO 2010 · 58:542-542

DOI 10.1007/s00106-010-2135-5

Online publiziert: 29. Mai 2010

๑) Springer-Verlag 2010

P.K. Plinkert - M. Praetorius

HNO-Klinik, Universitätsklinikum Heidelberg

\title{
Ausgezeichnet in HNO
}

sem Gebiete lastenden Vorurteile beigetragen zu haben!"

Im Jahr 2009 teilten sich Frau Prof. Dr. med. Nicole Rotter, HNO-Klinik der Universität Ulm, und Herr Prof. Boris Stuck, HNO-Klinik des Universitätsklinikums Mannheim der Universität Heidelberg, den Preis.

Der jährlich von der Deutschen Gesellschaft für Hals-Nasen-Ohren-Heilkunde, Kopf- und Halschirurgie vergebene Dissertationspreis ist nach Herrn Prof. Dr. Dietrich Plester benannt.

Aus Essen stammend erhielt er nach ethnographischen Studien in Afrika und pharmakologischer Ausbildung in Marburg seine HNO-Fachweiterbildung bei Wullstein in Siegen, um dann als international renommierter Ohroperateur an die Düsseldorfer HNO-Klinik zu Prof. Meyer zum Gottesberge zu wechseln. 1966 übernahm er den Lehrstuhl in Tübingen und machte die HNO-Klinik zu einem international bedeutenden Zentrum der Mikrochirurgie des Ohres und war lange Zeit der herausragende Repräsentant der deutschen Otologie weltweit. Zudem gründete er das „Tübinger Innenohrlabor". Sein Forschungsschwerpunkt waren neben der hörverbessernden Chirurgie einschließlich Keramik- und Goldimplantaten die Autoimmunologie des Innenohrs. „Plester-Prothese“ und „PlesterMesser" wurden international bekannt und tragen heute seinen Namen. Gemeinsam mit Prof. Rosen konnte er eine zivilisationsbedingte Komponente der Alterschwerhörigkeit aufzeigen. Eine große Zahl deutscher und ausländischer Ärzte besuchte seine jährlichen Kurse zur Mittelohrchirurgie. Seine Werke „Der Operationskurs des Hals-, Nasen- und Ohrenarztes" sowie sein "Atlas der Ohrchirurgie" wurden Standardwerke der HNOChirurgie.
Im Jahr 2009 wurde der Plester-Dissertationspreis an Herrn Dr. Andreas Brandt, HNO-Klinik des Universitätsklinikums Göttingen, zuerkannt.

Der jährliche Innovationspreis der Arbeitsgemeinschaft deutschsprachiger Audiologen und Neurootologen (ADANO) der Deutschen Gesellschaft für Hals-Nasen-Ohren-Heilkunde, Kopf- und Halschirurgie wurde im Jahre 2009 Herrn Dr. Ernst Dalhoff, HNO-Klinik der Universität Tübingen, zuerkannt.

Alle 4 Ausgezeichneten stellen ihre der Preisverleihung zugrunde liegenden Forschungsarbeiten in diesem Heft vor. Wir hoffen, dass Sie etwas von der Faszination dieses breit gefächerten Abbilds der vielfältigen Forschungsfelder innerhalb unseres Fachgebiets mitnehmen können.
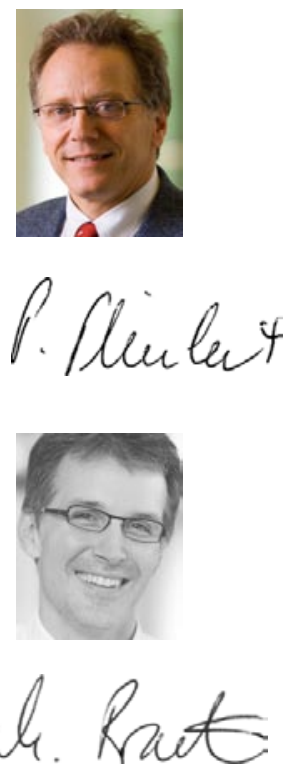

\section{Korrespondenzadresse Prof. Dr. Dr. h.c. P.K. Plinkert und PD Dr. M. Praetorius} HNO-Klinik, Universitätsklinikum Heidelberg Im Neuenheimer Feld 400, 69120 Heidelberg Peter.Plinkert@med.uni-heidelberg.de 